\title{
L'interprétation populaire de la maladie et de la guérison à lî̀le de la
} Réunion

Jacqueline Andoche

\section{Citer ce document / Cite this document :}

Andoche Jacqueline. L'interprétation populaire de la maladie et de la guérison à l'île de la Réunion. In: Sciences sociales et santé. Volume 6, n³-4, 1988. pp. 145-165;

doi : https://doi.org/10.3406/sosan.1988.1108

https://www.persee.fr/doc/sosan_0294-0337_1988_num_6_3_1108

Fichier pdf généré le 31/03/2018 


\title{
Resumen
}

Jacqueline Andoche : La interpretación popular de la enfermedad y de la curación en la isla de la Réunion.

En la isla de la Réunion, la interpretación de la enfermedad se hace la mayoría de las veces según una lógica proyectiva, que remite fuera de tema la causa del mal. La terapéutica apunta a restituir ese mal a su universo de origen (la naturaleza o el mundo de los espiritus) por expulsion o exorcisme

Esta manera de actuar, que traduce una relación con el afuera y con lo otro, permite, en una sociedad pluri-étnica nacida del encuentro entre diversas culturas, una lectura de las relaciones sociales. El perseguidor identificado se encuentra tanto más puesto en acusación como se diferencia culturalmente del grupo de pertenencia étnica de su víctima.

\begin{abstract}
Jacqueline Andoche: The Popular Interpretation of Illness and Cure in the Island of la Reunion. In the Reunion Island, disease is interpreted most commonly in terms of a logic of projection, which renders its cause irrelevant. Therapy is designed to return the disease to the world from which it originated (nature or the spirit world) through expulsion or exorcism.

This way of doing, which reflects a relation with another place and other people, enables a multi-ethnic society, originating as a meeting point for different cultures, to interpret social relations. The identified persecutor is ail the more accused because he is differentiated culturally and socially from the ethnie group of his victim.
\end{abstract}

\section{Résumé}

Jacqueline Andoche : L'interprétation populaire de la maladie et de la guérison à l'île de la Réunion.

A l'île de la Réunion, l'interprétation de la maladie se fait le plus souvent selon une logique projective qui renvoie hors sujet la cause du mal. La thérapeutique vise à restituer ce mal à son univers d'origine (la nature ou le monde des esprits) par expulsion ou exorcisme.

Cette manière de faire, qui traduit une relation à Tailleurs et à l'autre, permet, dans une société pluriethnique née de la rencontre entre cultures diverses, une lecture des rapports sociaux. Le persécuteur identifié se trouve d'autant plus mis en accusation qu'il se différencie culturellement et socialement du groupe d'appartenance ethnique de sa victime. 
Jacqueline Andoche*

L'anthropologie médicale en France a mis l'accent ces dernières années sur l'importance à accorder aux notions de sens et d'usages sociaux de la maladie (voir [1], [20], [21], [22]). D'importants travaux ont été réalisés dans cette perspective, aussi bien dans les sociétés lignagères d'Afrique que dans les domaines créoles (voir aussi [9], [10], [12]).

Cependant, à côté des Antilles, qui constituent un terrain privilégié d'étude, les îles de l'Océan Indien font figure de parent pauvre. A la Réunion par exemple, des descriptions ethnographiques de techniques traditionnelles de soins ont été effectuées par J. Benoist (voir [3], [4], [5], [7]). Les autres travaux anthropologiques sont des monographies de groupes ethniques, comme les Tamouls [2] ou les Musulmans Gujaratis [19], qui ne prennent pas la maladie pour objet spécifique d'étude. On trouve aussi des ouvrages de vulgarisation et des thèses de médecine portant sur les croyances et les pratiques magico-religieuses (voir [14], [15], [17], [181).

Pourtant, ici comme ailleurs, l'interprétation des faits de santé et de pathologie renvoie à une symbolique sociale : la société réunionnaise, vieille de trois cents ans, s'est constituée dans le cadre d'une histoire coloniale, par vagues de migrations successives venues d'Europe, d'Afrique, de Madagascar, d'Inde et de Chine ; depuis quelques années, immigrent aussi des travailleurs comoriens, qui ont le statut d'étrangers. Dans ce contexte pluriculturel, les relations entre les groupes ethniques, ainsi que les représentations qu'ils se font les uns des autres, sont dictées par la place qu'ils occupent dans la hiérarchie sociale et par le rôle qu'ils ont joué dans l'histoire de l'île.

* Jacqueline Andoche, anthropologue, 20 rue Morère, 75014 Paris. 
Ainsi, certains groupes marginaux et non encore intégrés parce qu'étrangers, comme les Comoriens qui forment dans les zones urbaines un lumpen - prolétariat vivant de petits métiers, sont objet de méfiance, de crainte, voire de rejet. Il en va de même pour d'autres groupes, comme les Tamouls qui ont participé à la mise en place de la société réunionnaise et qui sont aujourd'hui intégrés, mais qui l'ont été dans un contexte de rivalité culturelle face au pouvoir colonial. Immigrés au XIX $X^{e}$ siècle pour suppléer dans les plantations l'absence de main-d'œuvre due à la suppression de l'esclavage, les Tamouls demeuraient sujets de la Couronne Britannique. Ce statut d'étrangers leur a permis, contrairement aux anciens esclaves, de voir respecter leurs coutumes et leurs traditions, notamment religieuses, désignées en contre-partie par les missionnaires et les colons comme la source du mal par excellence. Etiquetées dès l'origine comme des manifestations du Diable et de la sorcellerie (1), les pratiques religieuses tamoules garderont dans les imaginations futures cette marque négative. Aujourd'hui, du fait qu'ils ont pu maintenir leur identité et leur "altérité ", les Tamouls intégrés comme les Comoriens étrangers sont considérés et craints comme porteurs de malchance et jeteurs de mauvais sorts (2).

Lorsqu'un malheur physique, psychique ou matériel survient, et à plus forte raison lorsqu'il perdure, les personnes soupçonnées sont, dans un premier temps, des proches, des "autres familiers " auxquels on attribue d'emblée l'origine de son malaise ou de son mal-être. Cependant, d'une façon générale, le persécuteur actif, celui qui œuvre à engendrer les maux ou la maladien, ne peut être qu'un "autre" socialement, culturellement, ethniquement différent : le sorcier malbar (3) ou comorien consulté par le parent proche devenu ennemi.

En ce qui concerne la maladie, ces représentations et ces attitudes renvoient à une logique sociale - puisque le persécuteur court d'autant plus le risque d'être mis en accusation qu'il occupe un statut d'étranger et une position marginale dans la société.

(1) Nous utiliserons dans cet article le terme "sorcellerie" dans le sens de "sorcery ": action des sorts.

(2) Il est à noter que d'autres groupes intégrés récemment (fin du XIX ${ }^{e}$, début du $\mathrm{XX}^{e}$ siècle), comme les musulmans Gujaratis et les Chinois, ne font pas l'objet de telles représentations, parce que, ayant immigré dans le cadre de la migration libre, ils n'ont pas entretenu les mêmes rapports avec le pouvoir colonial. De plus, loin d'être des marginaux, ils constituent une élite économique (commerçants).

(3) De “malabar ", c’est ainsi que sont désignés les Tamouls en créole. 
Elles organisent la pathologie selon un modèle projectif, la souffrance étant généralement interprétée comme le résultat d'un déséquilibre dû à l'action d'un agent extérieur, aussi bien lorsque la maladie est "diagnostiquée " comme naturelle que lorsqu'elle est attribuée à des sorts.

Pour une maladie considérée comme naturelle, on évoquera l'action de la nature (chaleur, soleil, froid...), les effets d'une mauvaise alimentation ou les conséquences d'un traumatisme ou d'un accident. Dans le cas d'un " arrangement " par les sorts, le malaise peut se manifester sous la forme d'une possession par une ou plusieurs entités envoyées à la victime par le jeteur de sorts : esprits de personnes mortes de morts non naturelles (suicide, noyade, accident, envoûtement) qui ne connaissent pas encore le repos éternel et vivent dans un monde proche de celui des vivants, divinités ou représentations religieuses des groupes désignés comme persécuteurs (" esprits malbars" ou " esprits comores").

Selon cette logique projective, les techniques de soins visent à rétablir l'équilibre détruit par l'agresseur extérieur en expulsant ou en renvoyant le mal à son milieu d'origine (nature, monde des esprits, lieu de culte d'appartenance) - ce qui peut être analysé comme une manière de gérer l'ailleurs et "l'autre ".

Dans les pages qui suivent, nous voudrions rendre compte de ces différentes interprétations de la maladie et de la guérison à l'île de la Réunion, en montrant en quoi, dans le cadre d'une société pluriethnique, elles traduisent des rapports sociaux.

Nous nous appuierons sur les résultats d'une enquête ethnographique réalisée dans le sud de l'île auprès de guérisseurs créoles et de pousaris, de malades et de familles de malades d'origine créole et tamoule, selon les méthodes classiques de l'ethnologie : entretiens non directifs, études de cas, recueils de biographies, observation directe et participante où est intervenue notre expérience de réunionnaise, petite-fille de guérisseur herboriste.

\section{I - La maladie vécue comme une rupture d'équilibre dont la causalité est externe}

Divers tableaux de la nosographie populaire montrent que la maladie est vécue comme une rupture d'équilibre dont la causalité est externe. 
Les refroidissements et les excès de chaleur

Dans une première catégorie de maladies, les maladies "froides" ou " chaudes", le déséquilibre est d'ordre thermique et se traduit soit par une déperdition de chaleur due à un refroidissement, soit par un excès de chaleur.

Le " refroidissement " est la conséquence de l'action du froid sur le corps considéré comme chaud. Il existe plusieurs formes de refroidissement, considérées comme plus ou moins graves selon que l'action du froid est externe ou interne, c'est-à-dire selon qu'elle ne se fait sentir qu'à la surface du corps ou qu'elle pénètre l'organisme.

Dans la pensée des informateurs, les symptômes d'un refroidissement externe sont caractéristiques : "des maux de tête plus ou moins violents, une sensation de froid intense qui envahit le corps du patient, gagné par la fièvre et les frissons... Le sang, refroidi petit à petit, n'est plus en mesure de réchauffer l'organisme ". Ils traduisent la perte d'immunité de l'organisme sous l'action du froid. La toux, le rhume ou la grippe, indifféremment appelés "grippe", angine ou "mal de gorge", des courbatures ou des douleurs dans le dos sont le signe ou la conséquence de ce type de refroidissement, qui n'est pas très grave.

Les formes internes du refroidissement apparaissent plus dangereuses. Il en est ainsi de la " pleurésie » et du refroidissement du corps de la femme indisposée, qu'on appelle parfois " tuberculose ".

La «pleurésie» est occasionnée par la consommation de boissons ou d'aliments froids lorsque l'on a le corps échauffé par la marche ou par le travail. Dans ce cas, le froid qui pénètre à l'intérieur du corps agit directement sur le sang: il le « saisit » et le "glace ", arrêtant aussitôt sa circulation et par là-même l'apport de chaleur nécessaire à la vitalité de l'organisme. La " pleurésie " est un mal dont il faut se soigner immédiatement car l'action du froid à l'intérieur du corps est rapide, instantanée : il faut boire de l'urine - non sa propre urine qui, déjà refroidie, ne saurait être efficace - mais celle d'une autre personne. Faute d'un tel traitement, le malade meurt dans les heures qui suivent. Certaines personnes, dit-on, meurent même sur le coup, car leur organisme ne peut résister à l'agression du froid, dont l'action est très bien traduite dans le langage courant par le terme "saisir", qui signifie surprendre : le corps est "saisi ", le sang est "saisi ".

On retrouve les mêmes termes dans la description de la " tuberculose ": la femme indisposée doit soigneusement éviter de prendre des bains ou d'avoir un contact prolongé avec l'eau 
froide car pendant cette période, son corps est plus chaud qu'à l'ordinaire et le sang qui s'en écoule facilite l'agression par le froid, qui s'engouffre à l'intérieur de l'organisme et le prend d'assaut. Ce genre de refroidissement ne se guérit généralement pas. Selon certains informateurs, la malade meurt dans d'atroces souffrances en étouffant, après avoir "rendu " tout son sang refroidi et devenu noir. Proches et moins proches mettent souvent en garde contre ce type de refroidissement. Chaque informateur cite le cas de telle jeune fille ou de telle femme imprudente, morte de ne pas avoir suivi le conseil de ses aînés.

Dans les maladies dues à l'excès de chaleur, les informateurs distinguent également entre les maladies graves et celles qui le sont moins. "L'insolation " ou " coup de soleil à la tête " est considérée comme grave. L'exposition prolongée au soleil fait «bouillir» le sang du cerveau et éclater ses veines. Le malade meurt d'hémorragies nasales et de violentes migraines. Notons qu'ici le sang n'est plus " saisi » mais qu'il «bout ". Certaines personnes disent que le soleil " cuit le sang". Ces expressions traduisent bien l'idée d'une augmentation de la température humorale au-dessus du seuil de l'équilibre thermique d'un corps en bonne santé.

Les "échauffements» ou «inflammations " qui résultent aussi d'un excès de chaleur semblent cependant moins dangereux. Ces troubles peuvent être dus à un déséquilibre organique interne, mais, la plupart du temps, ils sont occasionnés par la consommation d'aliments qui " chauffent ", c'est-à-dire qui ont des propriétés réchauffantes ou irritantes, comme certaines plantes utilisées dans le traitement du refroidissement, l'alcool, les épices et les médicaments lorsqu'ils sont pris en trop grande quantité.

Comme le refroidissement, les échauffements peuvent être externes ou internes. L'échauffement externe se traduit par des gerçures de la peau ou des irritations, généralement au niveau des muqueuses, de l'aine et des aisselles. Un orgelet est un échauffement (on dit plutôt " une inflammation de l'oil »). Un aphte est un échauffement de la bouche. L'échauffement ou l'inflammation internes affectent le "dedans ", c'est-à-dire l'appareil digestif. Ils se manifestent par des brûlures d'estomac ou d'intestins. La constipation ou la dysenterie sont aussi caractéristiques de ce type d'échauffement : le sang "trop chaud " se mélange à l'urine et aux selles, car l'intestin est " cuit " par les épices ou l'alcool. Certains informateurs précisent que ce type d'échauffement concerne surtout les hommes, qui boivent beaucoup, bien que les femmes puissent également en souffrir.

Les notions d'excès se retrouvent également dans le discours populaire sur les troubles "biliaires". Ici cependant, la maladie 
semble relever davantage d'un désordre humoral que d'un déséquilibre thermique. Pour décrire les troubles biliaires ou dus à un excès de bile, on dit que le patient "a la bile ", que sa bile "lève " ou "monte". Dans la "jaunisse", la bile trop abondante se mélange au sang et colore le corps du patient qui devient «tout jaune ". Cet excès peut provoquer une " révolution" qui se traduit par des vomissements incessants.

Si la bonne santé implique la circulation régulière du sang chaud à l'intérieur du corps, la manifestation de la bile, a fortiori lorsqu'elle se mélange au sang, s'avère pathologique. Ce désordre intra-organique, indépendant de la volonté et du contrôle du patient, est admirablement mis en évidence par les expressions "monte", "lève " et "révolution". L'évocation fréquente d'une causalité externe, comme la consommation importante d'aliments acides, "amers " ou alcoolisés, accentue le caractère " incontrôlable » de la bile en mouvement, introduisant un rapport implicite entre exogénéité et organicité.

\section{Les crises}

Pour parler des déséquilibres d'ordre psychique ou neuropsychique, les informateurs font surtout appel à la notion de "crise".

A la Réunion, lorsque l'on dit d'une personne qu'elle " tombe crise" ou qu'elle "gagne crise", cela peut vouloir dire qu'elle est sujette à des manifestations émotives soudaines et parfois violentes. Dans ce sens large, la crise se rapporte au comportement habituel d'une personne sensible et nerveuse, dont on dit qu'elle a des "crises de nerfs". Elle évoque aussi l'expression d'une vive colère - on dit alors "crise de colère " ou "crise de rage ». Différentes manifestations d'un délire comme l'auto-agression ou l'agression vis-à-vis de l'entourage, la fugue ou l'errance, les hallucinations, peuvent aussi être appelées "crises".

Dans une acception plus restreinte, celui qui " tombe crise" peut être épileptique, hystérique, avoir des convulsions ou tout simplement des crises de tétanie. Une simple absence proche des formes du "petit mal» de l'épilepsie est ainsi une "crise", de même que la réaction à un traumatisme physique ou affectif - les informateurs parlent alors de «crise de saisissement ".

Le saisissement est un mal qui occupe une place importante dans la nosographie populaire. Réaction à un choc physique ou psychique, il est considéré comme dangereux car il peut tuer ou rendre gravement malade. Un saisissement mal soigné "porte sur les nerfs", dit-on; il fait monter la tension et les nerfs "se mettent 
en boule ", amenant le patient à crier et à se débattre. Parfois, tout son corps se raidit: c'est la crise. La crise de saisissement peut également se manifester par une absence plus ou moins longue. On dit alors que le malade " perd connaissance ", c'est-à-dire qu'il n'a plus sa conscience. Fréquemment, le malade sentant venir la crise dit qu'il va " tomber". Cette expression est souvent employée pour décrire la crise. Ainsi, d'un malade qui souffre de crises, on dit qu'il «tombe »; pour indiquer la fréquence de ses crises, on dit qu'il «tombe " tant de fois dans la journée.

Dans "l'épilepsie" - on dit indifféremment "épilepsie", " pilepsi ", " mal kadik » (de mal caduc) ou « crise » - le malade se débat, "roule par terre", "se mord la langue " et "bave " (dans ce cas, la salive est contagieuse et il faut éviter son contact). Quelquefois, le malade «perd connaissance». Ce mal est dû, selon les informateurs, à l'hérédité, à la sorcellerie ou encore au saisissement. Il est difficile de faire préciser la différence entre crise d'épilepsie et crise de saisissement. Pour certaines personnes, toutes les crises sont soit des crises de saisissement, soit une conséquence de la sorcellerie. De ce fait, considérer toute crise comme de l'épilepsie relève d'un abus nosographique qui, ignorant le discours des malades, a souvent fait dire au corps médical que l'épilepsie était "reine " à la Réunion. Ceci conduit jusqu'à une époque récente à soigner les personnes qui « tombent crise " comme des épileptiques et à leur attribuer de ce fait un statut qu'elles n'ont pas dans leur communauté.

L'hystérie, par contre, est bien spécifiée dans le discours des informateurs. C'est une maladie essentiellement féminine, et honteuse car elle révèle le besoin de rapports sexuels. Dans la crise d'hystérie, la malade a une sensation d'étouffement et ressent des "palpitations" insupportables qui la poussent à "crier". Là encore, on dit que ses "nerfs se mettent en boule" ou que "ses nerfs lèvent". Le mot "nerfs" cst employé dans un double sens, celui du dérèglement neurologique propre aux autres formes de la "crise" et celui d'un fort désir sexuel. Certaines personnes utilisent plutôt le terme de "sens»: d'une malade en crise d'hystérie, on dira qu'elle "a ses nerfs" ou "ses sens" qui « lèvent ". Notons que les expressions « lèvent ", "se mettent en boule " ou " avoir les nerfs » traduisent bien ici, comme dans la description des troubles biliaires, une perturbation due à un mouvement incontrôlable la bonne santé se définissant comme un état où les "nerfs" seraient en repos.

Les différentes manifestations de la "crise" prise dans un sens restreint - épilepsie, hystérie, crise de saisissement, etc. peuvent être rapportées sommairement à ce que les informateurs 
appellent «maladies des nerfs ». Elles se distinguent des formes du dérangement mental ou de la folie, pour lesquelles il n'existe pas de véritables tableaux nosographiques.

Pour décrire le dérangement mental, on utilise toute une série d'expressions qui traduisent essentiellement un état: d'une personne ainsi perturbée, on dira qu'elle a l'esprit ou la tête " dérangés " ou " chavirés", que "sa tête n'est pas bonne ", qu'elle est "piquée ", " toquée ", "timbrée", " pas juste »; quelquefois, dans un sens ironique et pour résumer le caractère immaturé de cette personne, on dira qu'elle n'est " pas cuit ». On emploie aussi les mots "fou " ou "fou-fou », " dingue ", "loufoque» et, plus rarement, « maboule».

Ce qui différencie la folie des maladies des nerfs c'est que le déséquilibre y est permanent et correspond davantage à quelque chose de "déplacé dans le cerveau ", ce que traduit bien l'expression "chaviré ". Ce dérangement est ce qui fait l'anormalité du fou, dont on dit qu'il «n'est pas une personne comme les autres". Les informateurs précisent bien qu'un malade qui " tombe crise " est normal: ce n'est qu'au moment de la crise qu'il est perturbé ; dans la vie quotidienne, à la différence du fou, il est " comme tout le monde".

La sorcellerie apparaît au premier rang dans l'étiologie des troubles psychiques ou neuro-psychiques. Si elle ne rend pas compte des seules maladies psychiques ou nerveuses - des troubles organiques peuvent être aussi interprétés en ce sens, notamment s'ils s'inscrivent dans une chaîne de malheurs (maladie ou mort d'un parent, pertes d'animaux domestiques, mauvaises récoltes...) - les crises se laissent plus facilement diagnostiquer comme la manifestation d'un arrangement par les sorts, surtout si elles surviennent à l'âge adulte. Mais la sorcellerie n'apparaît pas comme la seule cause des crises ou de la folie. Le saisissement qui est à l'origine de diverses sortes de crises est aussi fréquemment invoquée: il peut «chavirer » l'esprit de quelqu'un et le «rendre fou ".

L'hérédité peut également être évoquée. Mais, dans la mesure où elle va à l'encontre du modèle projectif qui attribue la maladie à un agent extérieur, cette étiologie est surtout mise en avant par des personnes non directement impliquées dans le malheur psychique (parent éloigné, voisin, ami...). Le malade et sa famille parleront davantage de saisissement ou d'arrangement par les sorts. S'ils en viennent à mettre en cause l'hérédité, ce ne peut être que dans le sens d'une fatalité qu'ils subissent et contre laquelle ils ne peuvent rien.

Tout se passe comme si le malade ne devait jamais être rendu 
responsable de l'événement qui survient pour détruire l'équilibre de sa santé. Il ne peut s'agir que d'un accident qui s'abat sur lui contre sa volonté, et dont la maîtrise lui échappe (action de la nature, dérèglement humoral, mouvement des nerfs, intervention de la "sorcellerie"). En témoignent les expressions employées pour rendre compte des différentes formes du vécu pathologique, que nous tenterons de classer en deux groupes syntaxiques, qui sont aussi des unités de sens.

Dans le premier groupe, nous placerons les tournures grammaticales employant la voix passive, car elles présentent la maladie comme subie par le sujet. Ainsi, d'un patient ensorcelé, on dira : «il est arrangé » ou «il a été arrangé ». D'un malade atteint de saisisscment, on dira: "il est saisi " ou "il a été saisi " comme on dit aussi du corps ou du sang qu'ils sont "saisis par le froid ". Ces métaphores disculpent le sujet malade dans la mesure où elles permettent de localiser ailleurs, hors de lui, la source de sa malchance.

A ce premier groupe peuvent se rattacher les expressions construites autour du verbe "gagner" (gagn') - qu'on peut traduire par "est atteint de " ou «a contracté " - qui signifient bien que le malade subit une action extérieure : être atteint de saisissement ou de crises se dit en créole "gagn' saisissement ", "gagn' crise " ou "tomb' crise". De même, contracter un coup de soleil ou un refroidissement se dira " gagn' coup de soleil " ou "gagn' refroidissement $»$.

Dans le deuxième groupe, nous comprendrons les formes verbales conjuguées au présent ou au passé actif, qui mettent davantage l'accent sur l'action incontrôlable d'un agent pathogène et sont surtout utilisées pour décrire les désordres organiques ou humoraux. Ainsi, dans la "révolution de bile", c'est la bile qui "lève " ou "monte". Dans la crise de nerfs ou dans la crise d'hystérie, ce sont les nerfs qui "lèvent " ou " se mettent en boulc". Ccs formes verbales peuvent aussi être utilisées pour rendre compte d'un arrangement par les sorts. Dans ce cas, au lieu de dire «le patient est " ou "a été arrangé ", on dira : "d'mond' l'a aranz' à li ", ou encore "l'a met' sorcié d'su li ", ce qui permet de désigner directement l'agent persécuteur et responsable : «d'mond' ", «il ", " on ", c'est-à-dire le sorcier ou un ennemi pour le compte duquel travaille ce dernier - les expressions "d'mond' l'a aranz' à li » et "l'a met' sorcié d'su li " pouvant se traduire par: " on lui a jeté un sort ".

Dans une maladie dite "arrangée ", le déséquilibre organique ou psychique se double d'un déséquilibre dans l'ordre de l'univers qui entoure le malade. Celui-ci peut avoir des sensations 
d'étouffement ou de faiblesse qui s'ajoutent aux manifestations du mal dont il souffre. Il peut sentir que quelqu'un le touche ou encore, et c'est le cas le plus fréquent, entendre des bruits "bizarres" (bruits de chaîne, sons de cloche, courses d'animaux autour de sa maison...). Ces manifestations qui se produisent généralement la nuit sont le signe de la submersion par le surnaturel. Seul le «travail » du désenvoûteur ou de l'exorciste permet de rétablir l'équilibre des choses.

\section{II - La thérapeutique. Des techniques variées, une seule logique : l'exorcisme}

Dans sa démarche pour retrouver l'équilibre psychique ou somatique, le malade dispose d'un large éventail de soins. Le recours à la médecine moderne n'est pas négligeable. Dire qu'il n'intervient que lorsque le patient a épuisé les remèdes traditionnels relève d'une généralisation hâtive. L'interprétation de la maladie et l'itinéraire thérapeutique qu'elle implique dépendent des circonstances qui entourent la vie de chacun. Souvent, comme l'a observé J. Favret-Saada pour la France rurale [16], c'est un "annonciateur" qui amène le malade à s'interroger sur l'origine de ses troubles et à consulter un désenvoûteur. Cet annonciateur est généralement un proche : parent, voisin ou ami, qui a pu vivre dans le passé la même expérience pathologique et se charge de faire admettre une réalité difficilement acceptable. Entre le début de sa maladie et la consultation d'un désenvoûteur, le malade peut avoir recours aux différents agents de la médecine moderne : généralistes, spécialistes, hôpitaux. Cette démarche, qui peut aussi être parallèle à la consultation d'un ou de plusieurs guérisseurs, n'est nullement vécue comme contradictoire. Elle est fréquente pour les troubles d'ordre psychiatrique, où un mouvement de va-et-vient s'instaure entre l'hôpital ou le dispensaire et la maison du leveur de sorts. La venue à la psychiatrie peut aussi parfois se faire en dernière instance.

Dans le secteur de la médecine traditionnelle, les malades font généralement appel à des herboristes pour traiter une maladie organique ou nerveuse. La plupart des gens savent cependant comment préparer une "tisane" contre le refroidissement ou contre le saisissement.

Si la maladie est interprétée comme le résultat d'un arrangement, le patient est amené à faire appel à des techniques plus complexes. Les spécialistes en sont légion; ils peuvent être classés selon les techniques qu'ils utilisent ou selon la tradition à laquelle ils appartiennent, mais aussi selon leur réputation, qui correspond 
généralement à la place qu'ils occupent dans la hiérarchie des jeteurs de sorts. Ainsi, un désenvoûteur est considéré comme "fort " lorsqu'il peut aussi bien « tirer » que " mettre le sort " : plus il est craint, plus également son "travail " de désenvoûtement est jugé efficace.

Les exorcistes toutefois ne sont pas appréhendés en fonction de leur réputation. Prêtres ou laïques, ils procèdent à partir du Rituale Romanum ou du livre des Grands Exorcismes et bénéficient de l'aura protectrice de la religion officielle. Leur aptitude à " tirer " les sorts vient, dit-on, d'une longue habitude à côtoyer et à maîtriser les forces du mal.

Les prêtres tamouls, appelés « pousaris », sont aussi sollicités en matière de désenvoûtement. Là n'est cependant pas leur fonction première. Le "pousari ", officiant dans une chapelle, a la charge de veiller à l'organisation et au respect du culte. Il peut avoir des connaissances médicinales et, dit-on, " tirer" ou " mettre " les sorts. Il détient généralement ce savoir d'une initiation auprès d'un maître qui peut être un parent.

Entre les exorcistes et les pousaris, il existe toute une foule de thérapeutes qui pratiquent une magie syncrétique, aux frontières de la tradition catholique et de la tradition indienne. On les appelle " devineurs " ou "guetteurs ", car ils diagnostiquent l'origine de la maladie en la "guettant ", c'est-à-dire en regardant dans les cartes ou dans la cendre, ou en la "devinant " par voyance. Le devineur détient son don d'un Saint Patron avec qui il " travaille " et qui le protège. Ce maître est le plus souvent un saint catholique, St-Expédit; il peut être aussi une figure pieuse de l'histoire locale ou une divinité indienne. Il arrive que le devineur améliore ses connaissances par la lecture de livres « secrets " comme le Petit et le Grand Albert ou Le Dragon Rouge, ce qui ne va pas sans consolider malgré lui sa réputation de sorcier.

Exorcistes, pousaris et devineurs sont les thérapeutes locaux spécialisés dans le traitement de la maladie " arrangée ». Ils appartiennent à la tradition réunionnaise. Mais, depuis une quinzaine d'années environ, ils se voient concurrencés par les Comoriens qui s'installent dans l'île - notons que tous les Comoriens ne sont pas des désenvoûteurs, mais leur côté marginal et nomade (ils vivent pour la plupart du colportage) s'ajoute à leur statut d'étranger pour renforcer les stéréotypes dont ils sont l'objet. Ils sont réputés "grands sorciers", et les arrangements par leurs sorts irrémédiables s'ils ne peuvent être défaits par un des leurs ou par un " plus fort ". En ce sens, ils se substituent dans l'imaginaire local aux "malbars" qui ont occupé longtemps la première place en matière d'envoûtement et de désenvoûtement. 
Quelle que soit leur réputation ou leur tradition, les désenvoûteurs appliquent tous une seule et même logique thérapeutique, celle de l'exorcisme, qui consiste à "tirer ", à « faire sortir " du corps du patient le mal qui le fait souffrir et dont la présence est symbolisée par un «esprit " ou par des matières insolites que le malade "rend" pendant la cure. Le thérapeute diagnostique d'abord l'origine naturelle ou arrangée de la maladie en utilisant diverses techniques : transe, "regardage " dans la cendre ou dans les cartes, prières, etc. Puis il recherche l'agent de l'envoûtement (parent, ami ou voisin, envieux ou jaloux, amant déçu, etc.), ainsi que les moyens utilisés : drogue introduite dans la nourriture ou dans la boisson, bijoux offerts en cadeaux, "arrangement " par photos de la victime, objets maléfiques laissés dans sa cour, qu'il faudra retrouver et dont il faudra se débarrasser, soit en les jetant à la mer, soit en les apportant chez le désenvoûteur pour qu'il «travaille» dessus.

Pendant la cure, le patient peut être frappé pour chasser de son corps l'esprit qui le possède. On peut lui faire boire une tisane de plantes aux pouvoirs conjurateurs, réciter des prières ou lui imposer les mains en enjoignant au mal de sortir. Ces différentes techniques sont souvent associées. Les exorcistes utilisent l'eau et le sel bénits. Le sel bénit, introduit dans les yeux et la bouche du malade, fait fuir l'esprit rebelle. Certains devineurs opèrent par transfert de la maladie: pendant la cure, ils "prennent " le mal de leur client, c'est-à-dire qu'ils le vivent par simulation, puis le restituent à son milieu d'origine, le monde surnaturel. Cette technique nécessite, pendant les jours qui précèdent le désenvoûtement, une mise en condition du malade et du thérapeute. Le thérapeute doit prier, jeûner et prendre des bains rituels, afin d'être en état de pureté au moment du "travail». Au patient, il est souvent recommandé de suivre un régime végétarien, ce qui témoigne de l'influence de la tradition indienne sur la médecine syncrétique des devineurs.

La cure terminée, le désenvoûteur donne des conseils prophylactiques, dont celui d'éviter tout contact avec l'auteur présumé de l'arrangement. Il peut prescrire à son client des bains rituels, à prendre à midi ou à minuit et dont l'eau et les résidus seront jetés à la mer ou dans une "croisée de chemins "- lieux et moments qui sont, dans l'imaginaire local, privilégiés par les esprits, auxquels il faut restituer les forces par lesquelles ils dérangent pour rétablir l'équilibre entre le monde des vivants et celui des forces occultes.

Le thérapeute peut aussi confectionner une "garanti ", c'està-dire une amulette que le malade devra toujours porter sur lui 
afin d'éviter les rechutes. Enfin, les prières et les neuvaines de messes pour le repos des "âmes délaissées " sont toujours recommandées, de même que des offrandes au saint ou à la divinité avec lesquels "travaille " le désenvoûteur.

Si la logique de l'exorcisme se donne davantage à voir dans le traitement de la maladie arrangée, elle n'en est pas pour autant absente de celui d'une affection organique ou diagnostiquée comme naturelle : comme on "tire " l'esprit, on " tire " le coup de soleil, on "fait sortir " le refroidissement ou l'échauffement et le saisissement lui-même n'échappe pas à cette logique extractive.

Pour «tirer le coup de soleil", on prend un verre d'eau que l'on déplace sur la tête du patient. A l'endroit où se localise le " coup de soleil ", on voit des bulles dans le verre : c'est le signe que le mal s'en va. On arrête l'opération uniquement lorsque les bulles disparaissent. Cette technique peut être complétée par des opérations à caractère magique, comme la récitation de prières secrètes, l'évocation de la date de naissance du malade pendant la réalisation du "travail", une bougie allumée en face du patient pour symboliser la chaleur qui attire à l'extérieur le "coup de soleil ". Certaines personnes utilisent une feuille de chou ou de bananier dont les propriétés "rafraîchissantes " ont pour effet d'extirper l'excès de "chaud". Au bout d'un certain temps, le végétal apparaît " comme grillé ", c'est le signe que le "coup de soleil " a disparu.

Une technique rafraîchissante voisine est utilisée pour traiter l'échauffement interne: on applique sur le ventre du malade des cataplasmes de plantes qui, ayant cette propriété, attirent à l'extérieur la chaleur du corps et atténuent ainsi la douleur. Lorsque les feuilles sont "cuites ", on les remplace jusqu'à ce que le mal disparaisse. Dans le même temps, le malade boit des tisanes "rafrâ̂chissantes ", préparées par macération dans de l'eau froide ou en décoction, qu'on laisse d'abord refroidir car il ne faut pas ajouter à la chaleur d'un corps déjà " enflammé ".

Ainsi, dans le traitement du coup de soleil comme dans celui de l'échauffement, le rétablissement de l'équilibre thermique du corps se fait par l'attraction du mal "chaud" par un contraire "froid" ou "frais": eau ou plantes rafraîchissantes. S'il arrive qu'un élément de qualité "chaude"soit utilisé, comme la flamme de la bougie qui attire à l'extérieur le "coup de soleil ", cela ne contredit nullement la logique thérapeutique : il s'agit d'un moyen symbolique utilisé pour restituer plus efficacement la chaleur à son milieu naturel, l'élément thérapeutique principal étant l'eau "froide " du verre déplacé sur la tête du patient.

Dans le traitement du refroidissement, l'élément attractif est 
le " chaud ": il s'agit de réchauffer le corps et de le faire transpirer pour "faire sortir" le mal "froid". Le malade boit des tisanes "chauffantes", préparées à base de plantes comme la cannelle, le benjoin ou la citronnelle (4). Il peut prendre des bains et l'on frotte son corps à l'aide d'onguents faits d'alcool, de camphre ou de girofle, qui sont des éléments réchauffants. Jusqu'à une époque récente, la technique la plus usitée était l'application de ventouses, préalablement chauffées, sur le dos du patient: elles attiraient, disait-on, le mal vers l'extérieur. Dans le cas de refroidissement grave, on avait recours à des ventouses scarifiées: les premières ventouses étaient retirées pour effectuer des petites saignées sur le dos du malade, où des ventouses réchauffées étaient de nouveau appliquées. Cette opération permettait, selon les interlocuteurs, d'épurer le sang «saisi » par le refroidissement.

Dans le traitement du saisissement, on procède davantage par relation analogique avec la maladie : on utilise des plantes aux vertus calmantes et tranquillisantes, telles la marjolaine, la sensitive ou le romarin (5), sur lesquelles est réalisé symboliquement un transfert de la maladie. On jette les plantes avec du sel dans un récipient préalablement chauffé. Lorsque les ingrédients sont bien "roussis ", on verse rapidement de l'eau dessus, et on "étouffe " la tisane à l'aide d'un couvercle que l'on garde toujours à portée de la main. L'essentiel est d'agir rapidement car il faut "saisir" la préparation comme le mal a "saisi » le patient. Cette opération de " saisissement " ou "d'étouffement " est l'acte indispensable à la guérison (le sel a pour propriété de faire «fondre " le sang saisi par le traumatisme et les plantes calmantes atténuent l'anxiété du patient).

Certaines personnes utilisent des éléments étrangers, poussins ou escargots vivants, qui sont de véritables objets de transfert et qu'elles jettent dans la préparation au moment de son ébullition, après quoi elles "étouffent " la tisane. Lorsque celle-ci est prête, il faut toujours attendre qu'elle se refroidisse d'elle-même pour la boire: il ne faut jamais souffler dessus ni la mettre au frigidaire, car le froid artificiel pourrait de nouveau la saisir, ce qui aggraverait la maladie.

Cette opération de transfert, qui rappelle la technique utilisée par certains devineurs lorsqu'ils "prennent sur eux " la maladie de leurs clients, ne contredit pas la logique exorcistique. Lorsqu'on leur demande si saisir ou étouffer le saisissement n'équivaut

(4) Benjoin (Terminalia bentzoe - I - L.P) - Citronnelle (Lippia citriodorata).

(5) Sensitive (mimosa pudica linné). 
pas à le «faire rentrer ", les informateurs répondent catégoriquement : "On ne fait pas rentrer le saisissement, on étouffe la tisane pour que celle-ci soit plus efficace " - «Faire rentrer " est en effet une opération qui s'avère particulièrement dangereuse et qui ne relève généralement ni de la volonté du thérapeute, ni de celle du patient. Un malade atteint de rougeole qui commettrait l'imprudence de sortir de sa chambre "refroidirait " et ce refroidissement "ferait rentrer" la rougeole; or la disparition de celle-ci serait mortelle, car aucune action n'apparaîtrait plus alors possible.

La difficulté à se représenter une opération qui consisterait à insuffler un quelconque élément thérapeutique correspond bien à la logique projective, selon laquelle il s'agit de rétablir l'ordre des choses en restituant le mal à son milieu d'origine : "l'ailleurs" que cet «ailleurs » soit symbolisé par la nature qui «échauffe » ou "refroidit " et contre laquelle on se protège, qu'il soit figuré par les traumatismes physiques ou affectifs qui peuvent "saisir" le malade, qu'il soit représenté par le monde des "esprits " manipulé à l'encontre de la victime ou par l'étranger "grand sorcier " qui dérange par sa différence ou sa marginalité. Remise "en ordre » qui permet de faire l'économie d'une remise en cause de soi-même et, au-delà, de la hiérarchie sociale établie.

\section{III - L'interprétation de la maladie et de la guérison comme lecture des rapports sociaux}

Analyser le sens ou l'usage social de la maladie et de la guérison, dans une société donnée, c'est aborder le problème de l'efficacité symbolique et idéologique des représentations et des pratiques relatives aux événements pathologiques dans cette société. Si la question de l'efficacité symbolique renvoie à l'interprétation par les Réunionnais de la maladie selon le modèle exclusif de la projection, celle de l'efficacité idéologique se donne davantage à voir dans ce que $M$. Augé, à l'instar de Castoriadis, a défini comme la "nécessaire aliénation à l'institution" [1]: la soumission des individus - ainsi que des événements qui surviennent dans leur histoire, en l'occurrence la maladie - à un " imaginaire social » qui leur préexiste et qui totalise les interprétations mises en œuvre pour les comprendre et les maîtriser. A la Réunion, cette aliénation à l'institution se réalise en premier lieu dans l'application du code des relations aux autres. C'est elle qui donne sens au modèle projectif/persécutif.

Dans cette petite île montagneuse où le cloisonnement régional et la diversité ethnique ont favorisé de tout temps la 
constitution d'isolats, il existe plusieurs catégories de «l'autre", du plus au moins "semblable".

Cet autre peut être le parent - ascendant, descendant, collatéral ou allié - avec qui l'on entretient des relations dans le cadre quotidien des fréquentations de parenté et de voisinage. Comme dans la plupart des sociétés "traditionnelles", ces relations sont de dépendance mais aussi de rivalité. L'essence de l'individu se définit par rapport au groupe familial auquel il appartient : c'est celui-ci qui permet de l'identifier, qui peut éventuellement l'aider en cas de besoin, mais qui lui fait en revanche subir ses normes. Cette «solidarité mécanique" se distend en cas de conflits, aussi anodins soient-ils: lorsqu'une discorde éclate, le parent allié devient persécuteur, réel ou imaginaire. Objet de l'agressivité ou de la méfiance, il sera le premier soupçonné ou désigné comme responsable de la maladie ou du malheur qui s'abat par hasard sur soi. La plupart des histoires de sorcellerie sont rétrospectivement expliquées comme la conséquence de conflits familiaux, nés de jalousies ou d'antagonismes latents. Dans le cadre restreint de la parenté, l'autre est aussi le semblable, puisqu'il appartient à la même lignée, et habite souvent le même quartier ou le même village. Sa mise en cause, comme persécuteur et "promoteur " de la maladie et du malheur, permet de supporter l'emprise de l'idéologie familiale.

L'autre ressemblant, c'est aussi le voisin, celui qui n'est proche ni par le sang ni par l'alliance mais qui habite le même quartier ou le même village. Avec lui les relations sont aussi inévitablement à la fois hostiles et cordiales.

Cependant, l'autre par excellence, c'est l'étranger qui n'appartient ni à la même famille, ni au même village, ni à la même ethnie - celui qui n'est pas «du pays». Notons que dans cette société où dominent les relations de type communautaire, l'impossibilité d'identifier l'origine familiale d'un individu, même s'il est facile d'identifier son origine ethnique, fait de lui un étranger. L'habitant du village voisin, le plus proche soit-il, sera ainsi désigné. Avec lui les relations seront distantes et codifiées; du fait qu'on ne sait pas «qui " il est, cet inconnu devient une source potentielle de malheur: il peut voler, empoisonner ou même ensorceler. Cette méfiance trouve son illustration dans des conseils devenus proverbiaux, tels que : "il ne faut pas ouvrir sa porte à n'importe qui ", "il ne faut pas manger, il ne faut pas boire avec ou chez n'importe qui ". Certaines personnes refusent ainsi toute invitation en-dehors de la famille. Le code de politesse est luimême régi par cette réserve: lorsque l'on reçoit, il faut toujours ouvrir les bouteilles devant les invités; de même, on ne se permet- 
tra jamais de franchir le portail ou les limites de la cour d'une famille à qui l'on rend visite sans avoir été invité à "rentrer".

Ces comportements prennent sens dans les croyances aux sorts, puisque l'on peut envoûter par " empoisonnement ", c'est-àdire en « arrangeant » la nourriture ou la boisson que l'on offrira à sa victime. On peut aussi jeter un objet maléfique dans sa cour, qui est le lieu de sa vie privée, le prolongement de son intimité et qui, dans une société d'interconnaissances où l'individualisme n'est pas de bon aloi, permet de distinguer les membres d'un réseau de parenté et, au-delà de la parenté, les familles ou les personnes qui habitent le même quartier, voire le même village. Déposer les restes d'une cérémonie magique dans la cour ou devant le portail d'une famille ou d'une personne, c'est la toucher au plus profond de son être et lui signifier sa volonté irrémédiable de la détruire.

Ce code de relations présente cependant une marge de tolérance qui varie en fonction du statut de "l'étranger" et de son intégration à la société globale. Lorsque cet «étranger » est différent du simple fait qu'il n'appartient pas à la famille, au village ou à l'ethnie, il n'est qu'objet de méfiance: avec lui, on garde ses distances. Par contre, lorsqu'il s'affiche dans sa différence culturelle, sociale, historique, voire politique, il devient le symbole même de l'altérité et donc de l'adversité. Deux groupes ont occupé historiquement cette position dans la société réunionnaise: les «Malbars " et les «Comoriens".

Un regard sur la situation historique montre comment, au $X I X{ }^{e}$ siècle, les "malbars" sont des étrangers qui vont s'intégrer petit à petit à la société locale. Lorsqu'ils arrivent à la Réunion, les cadres de la société coloniale, vieille de deux siècles, sont bien établis. Au sommet, un petit groupe de colons d'origine européenne forme une aristocratie foncière qui se démarque de la masse des autres "blancs", pauvres en raison du manque de terres propices à l'extension du système de la plantation. Ces « pauvres blancs " forment, avec les métis et les esclaves affranchis, la majorité de la population.

La population d'origine africaine était relativement moins importante qu'aux Antilles, puisqu'à la Réunion les esclaves étaient originaires de la côte est de l'Afrique, mais aussi de Madagascar et déjà, dans une certaine mesure, de l'Inde; elle a donc moins pu manifester une solidarité " native". Réifié, christianisé, l'Africain n'a pu sauvegarder sa culture face à l'acculturation et à l'assimilation forcées du système colonial. De ce fait, pour lui comme pour les autres groupes sociaux, la différence avec le maître blanc est essentiellement une différence de statut économique. L'engagé indien, immigré la plupart du temps avec sa famille 
et protégé par une législation étrangère, la loi britannique, voyait ses coutumes et ses traditions, marquées par l'hindouisme populaire sud-indien, respectées. Ces coutumes et traditions se sont quelque peu transformées en raison de l'exil, mais elles sont restées néanmoins spécifiques à l'intérieur de la société réunionnaise. Leur apport aux syncrétismes magico-religieux n'est pas négligeable, les pratiques des devineurs en sont un bon exemple.

Au départ cependant, cet étranger nouveau-venu, ce «coolie " comme on l'appelle alors, intrigue ou effraye par sa différence, d'autant que cette différence est dénigrée par le pouvoir colonial. Aujourd'hui encore, les activités du " pousari ", qui n'est souvent qu'un officiant, sont interprétées comme des actes de sorcellerie. De même, les "esprits" possesseurs, ceux qui rendent malade, persécutent, déséquilibrent, sont souvent des divinités indiennes, des " zespri malbar ". Lorsqu'un indien tamoul consulte un exorciste catholique, sa maladie est ainsi interprétée comme le résultat de ses pratiques maléfiques et la liste des « zespri malbar " qui sont censés le « travailler » est souvent fort longue.

Aujourd'hui les malbars sont bien intégrés à la société locale. Ce sont des Réunionnais à part entière. Certains font partie de l'élite politique et intellectuelle, d'autres ont accédé à la propriété foncière. Même s'ils gardent quelque peu la marque négative de leur différence, ils ne sont plus aussi craints que les "Comoriens", dont la sorcellerie, pense-t-on, peut tuer à distance. La technique de ces derniers, désignés comme les plus «forts » dans ce domaine, est considérée comme d'autant plus efficace qu'ils passent la mer pour aller chercher le sort dans leur pays. Les activités du « pousari » sont en comparaison moins redoutables et moins redoutées car il fait partie de la société réunionnaise, et il apparaît toujours possible d'opposer une action rivale ou contraire à son éventuelle action maléfique, qui trouve sa source dans le pays même. Tout se passe, dans cette société insulaire où le monde s'arrête aux limites de l'océan ou des montagnes qui bordent le village, comme si le mal ne pouvait trouver sa source que dans ce qu'il y a de plus éloigné, de plus extérieur, de plus différent.

Que cette différence soit celle d'un étranger noir, marginal de surcroît (paria ou prolétaire nomade), renforce le stéréotype dont il est l'objet, mais ne le constitue pas. Dans un cadre géographique peu propice au développement du système de la plantation, de son idéologie manichéiste et de ses oppositions noir $/ \mathrm{blanc}=\mathrm{mal} / \mathrm{bien}$, dans cette société coloniale où le passé africain est gommé et où le pouvoir ne se fonde pas sur la couleur de la peau mais sur la réussite et la richesse, l'étranger remplace l'africain absent. La phantasmatique dont il est l'objet permet, comme la persécution 
dans le milieu familial ou villageois, de ne pas remettre en cause un ordre social imposé par l'histoire et le consensus sur lequel repose la coexistence de groupes ethniques qui n'ont pas choisi de vivre ensemble.

Ainsi, participant à un modèle projectif qui transcende et marque les habitudes et les modes de vie, les représentations de la maladie et de la guérison prennent tout leur sens : vécu comme persécuteur du corps et de l'esprit dont il dérègle le fonctionnement, le mal, quelle que soit sa nature, doit toujours être expulsé ou exorcisé. Contre son agression, il existe un code de la prévention qui, comme celui des relations sociales, régit les rapports à l'ailleurs, aux mondes naturel et surnaturel où ce mal trouve sa source.

Contre le monde surnaturel, celui des esprits qui peuvent être manipulés à l'encontre des vivants, il existe tout un arsenal de protections. Les plus classiques sont la bénédiction des maisons et le port de "gardes-corps" de toute sorte, dont les médailles de St-Benoît ou de St-Georges, les chapelets et les scapulaires. Certaines personnes exposent des images pieuses à l'entrée de leur maison ou font construire des petits oratoires dans leur cour. Mais c'est surtout hors de chez soi et, en particulier, la nuit que l'on s'expose, dit-on, à des rencontres dangereuses. Aussi est-il conseillé d'éviter à certaines heures (six heures ou minuit) les lieux privilégiés par ces esprits : les cimetières, l'enceinte des églises, les croix de Jubilé, les "croisées de chemins " où les sorciers sont censés venir finir leur «sabbat", les alentours des chapelles indiennes et, enfin, la résidence de toute personne accusée ou soupçonnée de sorcellerie. Lors d'une sortie nocturne, on dispose de recours d'urgence en cas de mauvaise rencontre : se signer ou invoquer un saint protecteur, menacer l'esprit en l'injuriant, retourner sa veste ou son chapeau, marcher à reculons pour rentrer chez soi. Ces conduites inversées, qui rappellent la logique thérapeutique visant à faire sortir ce que le mal fait rentrer, ont pour but de dérouter l'esprit et de l'empêcher de suivre sa victime. Le port de sel sur soi ou l'usage d'eau bénite sont aussi des moyens efficaces de se protéger.

Quant à la prévention à l'égard de l'environnement naturel, elle prend souvent l'allure d'interdits rigoureux et catégoriques. Ainsi, " il ne faut pas" se baigner ou boire froid lorsque l'on a le corps chaud ou le sang en mouvement: pendant les règles, après une hémorragie, si bénigne soit-elle, après un dur travail ou une longue marche. De même, il est formellement déconseillé de s'exposer longuement au soleil. Si on se trouve dans l'obligation de le faire, "il faut" toujours se coiffer d'un chapeau ou porter une 
ombrelle. La rupture de ces interdits expose à des refroidissements ou à des coups de soleil mortels.

On trouve cette même logique préventive dans la société antillaise, comme le montre $\mathrm{C}$. Bougerol pour la Guadeloupe où l'environnement est aussi perçu comme hostile. Comme à la Réunion, "l'eau et l'air refroidissent, on craint leur action sur l'extérieur du corps et à plus forte raison leur intrusion dans celui-ci. Quant au soleil, il échauffe l'organisme » [11]. Les attitudes à l'égard du monde surnaturel sont semblables : «des objets portés sur soi - les protègements - des formules récitées à voix basse, des rituels effectués autour de la maison servent à éloigner les sorts et à se protéger des envoûtements " [11].

Plus que l'existence d'universaux, ces analogies montrent l'emprise exercée par une pensée médicale et religieuse dominante, celle des colons européens immigrés ici et là vers le XVII ${ }^{e}$ siècle, qui s'est maintenue dans des situations historiques quelque peu différentes, marquant les représentations populaires de la pathologie et de la thérapeutique et les rapports à la souffrance, où l'on peut lire les rapports sociaux.

\section{RÉFÉRENCES BIBLIOGRAPHIQUES}

[1] Augé (M.), Herzlich (C.), Le sens du mal; Anthropologie, histoire, sociologie de la maladie, Editions des Archives Contemporaines, Paris, 1984.

[2] Barat (C.), Des Malabars aux Tamouls: l'hindouisme dans l'île de la Réunion, Thèse d'Anthropologie sociale et culturelle, 3 volumes, E.H.E.S.S., Paris, 1980.

[3] Benoist (J.), "Médecins, malades et guérisseurs ", Environnement Africain, I.D.E.P. (Dakar), 1, 1975, pp. 43-69.

[4] Benoist (J.), Carnets d'un guérisseur réunionnais, St Denis, F.R.D.O.I., documents et recherches, $1980, \mathrm{n}^{\circ} 7$.

[5] Benoist (J.), "Rencontres de médecines aux Mascareignes ", Bulletin d'Ethnomédecine, novembre $1981, \mathrm{n}^{\circ} 7, \mathrm{pp} .5-15$.

[6] Benoist (J.), Religion hindoue et dynamique de la société réunionnaise, CERSOI, GRECO Océan Indien du CNRS, Presses Universitaires d'Aix-Marseille, 1981 .

[7] Benoist (J.), "Possession, guérison, médiation : un chamanisme indien à l'île de la Réunion ", L'Ethnographie, 2, 3' trimestre 1982, pp. 227-240.

[8] Un développement ambigu, structures et changements de la société réunionnaise, St Denis, F.R.D.O.I., documents et recherches, 1983, $\mathrm{n}^{\circ} 10$.

[9] Bougerol (C.), La médecine populaire à la Guadeloupe, Paris, Editions Karthala, 1983.

[10] Bougerol (C.), «Ensorcellement maternel et trouble mental aux Antilles: histoire d'un cas ", Sciences Sociales et Santé, vol. III, n 3/4, 1985, pp. 151-167.

[11] Bougerol (C.), «Logique de l'excès, logique de la rupture ", L'Ethnographie, LXXXI, n 96-97, 1985. 
[12] Bougerol (C.), “ Héritier de la malédiction ou de la persécution à la maladie mentale ", Psychopathologie Africaine, 20, n 3, 1987, pp. 245-271.

[13] Chaudenson (R.), Le lexique du parler créole, Paris, Champion, 2 volumes, 1974.

[14] Chaudenson (R.) ed., Encyclopédie de la Réunion. St Denis, Livres Réunion Editeurs, 6 volumes, 1980.

[15] Chaudenson (R.) ed., Magie et sorcellerie à la Réunion, St Denis, Livres Réunion Editeurs, 1983.

[16] Favret-Saada (J.), Les mots, les morts, les sorts, Paris, Gallimard, 1977.

[17] Guignard (D.), Si je dis sorcier, St Denis, Institut de linguistique et anthropologie, 1986.

[18] Mouls (G.), Etudes sur la sorcellerie à la Réunion, St Denis, Editions UDIR, collection Anghaing, 1982.

[19] Nemo (J.), La communauté Gujarati de la Réunion: Islam et poursuites commerciales, Thèse d'anthropologie, 2 volumes, E.H.E.S.S., Paris, 1980.

[20] Zempleni (A.), L'interprétation et les thérapies traditionnelles des désordres mentaux chez les Wolof et les Lebou du Sénégal, Thèse de doctorat, Université de Paris, 1968.

[21] Zempleni (A.), «Anciens et nouveaux usages sociaux de la maladie en Afrique ", Archives des Sciences Sociales des Religions, LIV, 1, 1982, pp. 5-19.

[22] Zempleni (A.), "La maladie et ses causes ", L'Ethnographie, LXXXI, n96$97,1985$. 